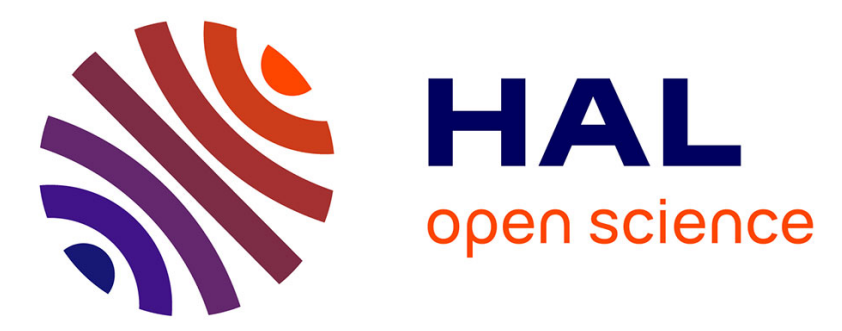

\title{
Le Père Lhande (1877-1957), pionnier de la prédication radiophonique
}

Corinne Bonafoux-Verrax

\section{To cite this version:}

Corinne Bonafoux-Verrax. Le Père Lhande (1877-1957), pionnier de la prédication radiophonique. Revue des Sciences Religieuses, 2004, 78 (3), pp.401-416. 10.3406/rscir.2004.3731 . hal-02512952

\section{HAL Id: hal-02512952 \\ https://hal.univ-smb.fr/hal-02512952}

Submitted on 20 Mar 2020

HAL is a multi-disciplinary open access archive for the deposit and dissemination of scientific research documents, whether they are published or not. The documents may come from teaching and research institutions in France or abroad, or from public or private research centers.
L'archive ouverte pluridisciplinaire HAL, est destinée au dépôt et à la diffusion de documents scientifiques de niveau recherche, publiés ou non, émanant des établissements d'enseignement et de recherche français ou étrangers, des laboratoires publics ou privés.

\section{(ㅇ)(1) $\$$}

Distributed under a Creative Commons Attribution - NonCommercial - NoDerivatives| 4.0 


\section{Le Père Lhande (1877-1957), pionnier de la prédication} radiophonique

Madame Corinne Bonafoux-Verrax

\section{Citer ce document / Cite this document :}

Bonafoux-Verrax Corinne. Le Père Lhande (1877-1957), pionnier de la prédication radiophonique. In: Revue des Sciences Religieuses, tome 78, fascicule 3, 2004. Prédication et prédicateurs. XIXe-XXe siècles. pp. 401-416;

doi : https://doi.org/10.3406/rscir.2004.3731

https://www.persee.fr/doc/rscir_0035-2217_2004_num_78_3_3731

Fichier pdf généré le 02/05/2018 


\title{
Résumé
}

La personnalité du Père Lhande est surtout connue pour son apostolat en milieu ouvrier dont des titres comme Le Christ dans la banlieue ou La Croix sur les fortifs témoignent fortement. Or, de 1927 à 1934 , le père jésuite est aussi le prédicateur le plus écouté de la radio et un véritable pionnier en matière de prédication radiophonique inventant un style parlé, voire des sermons dialogues avec ses auditeurs. Les nombreuses lettres qu'il reçoit permettent d'ébaucher les profils de ses auditeurs, de comprendre leur attente et ce qui leur plaît particulièrement dans ce nouveau média utilisé au service de la foi. De sa vaste tribune, le $\mathrm{P}$. Lhande lance aussi avec succès des appels à la charité au moment des grands froids de l'hiver ou des inondations.

\begin{abstract}
The personality of Father Lhande is essentially familiar to us on account of his apostolate in the working class ; books such as Le Christ dans la banlieue or La croix sur les fortifs are clear evidence for that. But, between 1927 and 1934, our Jesuit father was also the most popular preacher on the radio and a real forerunner in the field of radiophonie preaching, devising a spoken style of his own, even sermons in the form of a dialogue with his listeners. The many letters he received allow the author to outline the moral features of the listeners, to understand their expectations and to realise what they liked best in the new medium when used for the service of the faith. From his exceptional pulpit, Father Lhande also successfully appealed to the generosity of his listeners during the colder periods of winter or when floods occurred.
\end{abstract}


Revue des Sciences Religieuses 78 n³/2004, p. 401-416.

\section{LE PÈRE LHANDE (1877-1957): PIONNIER DE LA PRÉDICATION RADIOPHONIQUE}

Né à Bayonne d'une mère basque ayant vécu en Argentine, il passa son enfance dans le château familial et à Bayonne où il fit son grand séminaire. Admis dans la Compagnie de Jésus, il entra en 1900 au noviciat de Rodez mais fut obligé de le quitter un an plus tard. En effet, le P. Lhande appartient à cette génération de religieux contraints de quitter la France en 1901. Lors d'une conférence à Rodez, il a raconté, comment, quittant son pays rouergat, il se mit à genoux devant la portière du train et fit cette simple prière: "NotreDame de Rodez! Notre-Dame de Ceignac! un jour, rameneznous! »1

Après un long séjour en Espagne, le P. Lhande rejoint la communauté de la rue Monsieur le Prince à Paris qui compte à son arrivée notamment les Pères Henri du Passage, Yves de La Brière, Paul Doncœur, de Parvillez. La tâche qui lui est confiée aux Études est celle de la critique littéraire, mais un article publié le 5 septembre 1925 sur la détresse des mal lotis révèle une vocation missionnaire.

Les facettes de l'action et de la personnalité du P. Lhande sont multiples. Il s'agit d'un homme profondément enraciné dans la terre de son enfance. Sa connaissance et son amour du basque sont à l'origine d'un enseignement de langue basque à l'Institut catholique de Toulouse et de l'écriture de romans. Beaucoup sont inspirés par le pays basque comme Luis en 1912 ou Mirentchu, Yolanda et Mon petit prêtre dont les Études eurent le plus souvent la primeur. En 1926, il publie un dictionnaire basque-français et devient membre

I Discours du P. LHANDE au congrès diocésain de la Fédération nationale catholique, 25 septembre 1927, dans Bulletin de la Ligue des catholiques du Rouergue, septembre-novembre 1927, n 6, p. 14. 
de l'Académie de langue basque de Bilbao. Enfin, chaque été, il se ressource dans les montagnes basques où il passe quelques semaines avec un ami berger analphabète.

Deux aspects de l'action du P. Lhande sont assez exceptionnels: sa prédication à la radio et son engagement missionnaire dans les banlieues. De ce dernier qui ne constitue pas notre sujet, rappelons simplement que de ses visites méthodiques dans les banlieues, il tira la matière d'articles parus dans Études puis dans trois volumes: Le Christ dans la banlieue en 1927, Le Dieu qui bouge en 1930 et La Croix sur les fortifs en 1931. Il n'est, bien sûr, pas totalement indifférent au sujet que la prédication radiophonique s'incarnât durant les premières années dans une personnalité forte et particulièrement sensible aux souffrances des milieux populaires et ouvriers. Sa popularité est très grande: dès 1931, Le Christ dans la banlieue a connu des dizaines d'éditions successives. Le P. Lhande est un auteur lu, une enquête de la Ligue patriotique des Françaises auprès de ses adhérentes le cite au sixième rang des auteurs les plus lus.

Mais ici, c'est bien le prédicateur qui nous intéresse. Or, sa prédication se termina de façon brutale, l'orateur charismatique perdit la parole après une attaque foudroyante survenue en avril 1934. Son aphasie ne fut pas totale et il reprit de temps à autre le micro mais la parole ne lui était revenue que de façon partielle et difficile. Il vécut les vingt dernières années de sa vie retiré dans son pays natal à Tardets. Paul Doncoeur écrivit à sa mort: "Il est bouleversant de penser qu'à ce prêtre qui l'avait si bien servi, ce fut précisément la parole dont Il lui demanda le sacrifice $»^{2}$.

\section{La prédication radiophonique : une aire d'expression bien délimitée}

Il faut d'entrée de jeu tracer les limites de cette prédication, qui ne dispose pas d'une radio catholique, et rappeler que dans les années 1920 ce média est encore rare.

La France n'a pas inventé la prédication radiophonique, d'autres pays l'avaient précédée dans l'utilisation religieuse des ondes hertziennes et dans le développement général de la radiophonie. Il faut rappeler qu'à la fin des années 1920 , il y a 9 postes pour

2 «Le P. Lhande», dans Informations catholiques internationales, $1^{\mathrm{er}}$ mai 1957. 
100 habitants aux États-Unis, 6 en Angleterre, 1,5 pour la France 3. C'est surtout dans les années 30 que la radio se développe en France, passant d'un demi-million de postes récepteurs déclarés à près de 5 millions de postes en 1938-1939. Le réseau français compte 14 stations privées ${ }^{4}$ et 10 stations d'État. Le seul poste ayant un programme religieux régulier est celui de Radio-Paris, il a d'ailleurs le souci d'équilibrer ses émissions entre les trois confessions présentes en métropole. C'est ainsi que Radio-Paris octroie une causerie d'une demi-heure hebdomadaire aux protestants le jeudi, aux Israélites le vendredi ${ }^{5}$ et aux catholiques le dimanche. Par ailleurs, Radio-Paris diffuse les prédications des six dimanches du carême, le sermon du dimanche à Notre-Dame toute l'année et celui du pasteur Boegner à l'église réformée de Passy le vendredi. Les catholiques ont souvent tenté de conquérir une plage de temps supplémentaire. Ainsi, le P. Lhande écrit-il au secrétaire de la Fédération nationale catholique:

Il sera, je crois, impossible d'obtenir une série de conférences catholiques sur l'Éducation, car d'après les principes de la maison, il faudrait aussitôt introduire des conférences protestantes, israélites sur le même sujet. Les conférences du mercredi sur l'éducation laïque ont été établies comme compensation à la causerie catholique du dimanche. Toutes nos tentatives pour avoir une émission hebdomadaire en plus de celle-ci ont échoué 6 .

La menace de la suppression des émissions religieuses est une question pendante dès les débuts. Les catholiques connaissent le vœu du Congrès de la Libre pensée qui demande en 1927 leur suppression. Le $\mathrm{P}$. Lhande s'amuse à faire lire son sermon à Londres en langue française afin de démontrer que toute interdiction pourrait être tournée? . Pourtant dès que l'État acquiert le poste de Radio-

3 D'après un article du Correspondant: «La grande pitié de la radiodiffusion», 25 janvier 1930.

4 Dont Radio-Paris, Radio-Toulouse.

5 La causerie protestante est organisée par La Cause, dirigée par le Pasteur Durlemann, et la causerie israélite par La voie d'Israël du rabbin Algazi.

6 Lettre du P. LHANDE à Louis Leroux, Fédération nationale catholique, 9 mai 1931. Archives de la Société de Jésus à Vanves (ASJV), L5/1.

7 Récit dans la préface du Bon pasteur entièrement consacrée au problème de la liberté de parole sur les ondes (Paris, Spes 1928, p. 8). 
Paris, il passe à la réalisation d'un projet dont il sait d'avance qu'il va susciter de nombreuses réactions.

Le 17 décembre 1933, l'État entre en possession du poste national de Radio-Paris. Dans les semaines qui suivent, le ministre Mistler convoque Mgr Crépin, auxiliaire de l'archevêque de Paris, pour lui signifier, en attendant une décision du Président du Conseil qui est alors Camille Chautemps, la suppression des causeries catholiques du dimanche. On demande au P. Lhande de ne pas avertir les auditeurs, il s'exécute. Mais dès que la suppression est appliquée, les protestations affluent, la presse semble en cette circonstance défendre la liberté de ces causeries. Le gouvernement publie alors un communiqué précisant que le caractère exceptionnel des sermons de carême ainsi que le nombre très élevé d'auditeurs le conduisent à les conserver mais que les trois causeries hebdomadaires doivent disparaître afin de respecter la neutralité. Le Populaire après avoir revendiqué le droit de laïcité et l'exclusion du P. Lhande du micro de Radio-Paris ${ }^{8}$ est prêt à l'admettre de nouveau. À l'origine de ce revirement, l'ampleur de la protestation mais aussi l'inanité de la mesure. En effet, les causeries du P. Lhande ne se sont pas interrompues un seul dimanche. Il avait parlé pour la dernière fois à Radio-Paris le 31 décembre 1933 et le dimanche suivant 7 janvier par Radio-Luxembourg poste nettement plus puissant $(200 \mathrm{~kW}$ contre 75).

Une grande réunion de protestation est fixée au 7 février, les événements du 6 lui donnent des couleurs sombres. Cinq mille personnes écoutent les discours du général de Castelnau, de Jean Le Cour Grandmaison, du P. Lhande, du pasteur Durrleman et du rabbin Algazi. Le général de Castelnau constate l'échec complet de la tentative de laïcisation des ondes en France et même l'amplification de l'évangélisation catholique par une voix française à travers l'Europe. Il estime toutefois que cette situation, pour favorable qu'elle puisse paraître, ne saurait durer.

Nous ne pouvons pas admettre qu'on oblige une voix française éminemment réconfortante et moralisatrice à utiliser un microphone étranger [...] Nous ne devons pas tolérer l'état d'infériorité morale et même de diffamation que cette situation comporte vis-à-vis de la France aux yeux des étrangers?.

8 Article du 29 novembre 1933. 
Le gouvernement demande un rapport à la commission des PTT à la Chambre qui conclut que les émissions religieuses à la radio ne peuvent être prohibées car «l'athéisme à la différence de ce qui se passe en URSS n'est pas en droit français un principe d'ordre public $\gg 10$.

Il est à noter que la protestation de la Fédération nationale catholique inclut le droit de tous les croyants aussi bien «protestants, israélites, musulmans français ${ }^{11}$. Le ministre des PTT, André Mallarmé, rétablit les émissions religieuses dès le $1^{\text {er }}$ avril 1934, l'interruption a été de courte durée. Il a d'ailleurs demandé la création d'émissions d'ordre philosophique et moral confiées à des universitaires positivistes ou spiritualistes dont la première est assurée par Bergson.

Devant les incertitudes et les limitations, certains catholiques aspirent à la création d'un poste émetteur catholique: c'est le cas de Maurice Avril, qui ayant créé le Laboratoire d'études de TSF de Bretagne écrit au P. Lhande pour lui demander si cette idée lui parait réalisable ${ }^{12}$. Celui-ci estime que tant que Radio-Paris diffuse les causeries, il n'y a pas lieu d'entreprendre autre chose.

L'étendue couverte par l'émetteur Radio-Paris dépasse les frontières françaises et atteint la Belgique, l'Angleterre, le Maroc, l'Allemagne et d'autres pays encore. Les auditeurs francophones écrivent au P. Lhande ${ }^{13}$. Les différents volumes de correspondance semblent indiquer qu'au-delà des frontières de la France, le $P$. Lhande est surtout écouté en Belgique ${ }^{14}$.

Pour nous qui habitons une petite ville perdue près de la frontière allemande, c'est un plaisir d'écouter souvent Radio-Paris: nous croyons entendre l'âme de cette France que nous connaissons pour l'avoir parcourue et que nous aimons pour la bien connaître ${ }^{15}$.

\footnotetext{
9 Discours du général de CASTELnaU, 7 février 1934, salle Wagram. Papiers privés Castelnau «Fédération nationale catholique» carton 3, dossier 47-65.

10 Rapport présenté par Charles GUERNIER député d'Ille-et-Vilaine. Annexe au Procès verbal de la séance du 16 mars 1934.

11 Ibid.

12 Lettre de Maurice AVRIL du 27 novembre 1927 (ASJV L5/1).

13 « Extraits de correspondance » (ASJV L5/1).

14 Centaines de lettres d'auditeurs (ASJV L5/5.4).

15 Lettre du 20 mars 1929 (ASJV L5/5.1).
} 
Enfin, il n'est pas inutile de rappeler que l'Église n'éprouve aucune réticence devant ce nouveau vecteur de communication, contrairement au cinéma pour lequel son jugement est beaucoup plus critique. Elle voit dans la radio la possibilité de réaliser les paroles de l'Écriture sainte: «Allez et enseignez tous les peuples, prêchez l'Évangile à toutes les créatures»; "Leur voix est allée par toute la terre et leurs paroles jusqu'aux extrémités du monde $»^{16}$.

Le $\mathrm{P}$. Lhande lui-même consacre à la radio quelques-unes de ses causeries et si la radio peut, selon lui, à juste titre être qualifiée de don divin, c'est par son caractère mystérieux, spiritualiste et religieux ${ }^{17}$. Ce mystère tient selon le Père Lhande à l'aspect immatériel de la communication et qui fait qu'il est naturel, selon le P. Lhande, «que devant cette force fluide, intelligente et foudroyante, l'homme frappé d'une émotion infinie, se prenne à scruter, par-dessus le déconcertant spectacle de la terre, le mystère que nous cache le firmament. Je dis, de plus, que la radiophonie ramène à la voie de Dieu, parce qu'elle est la plus spiritualiste de nos découvertes ». Le P. Lhande signifie par là que même si la radio peut tout simplement distraire, elle excelle dans la haute éloquence, la prière et l'émotion. Enfin, elle est religieuse par elle-même, écrit le $\mathrm{P}$ Lhande, citant les témoignages de ses correspondants sur «l'impression de recueillement que produit dans l'atmosphère intime du foyer, cette voix grave d'homme venue de l'infini allant à l'infini ${ }^{18}$. Pour le $\mathrm{P}$. Lhande, la radio est donc beaucoup plus qu'un support neutre qu'il suffit d'utiliser. Il lui prête des vertus spécifiques, ce qui explique son enthousiasme pour ce type de prédication.

Toutefois, la radio ne saurait dispenser les fidèles d'aller à la messe et le Saint-Office rappelle à plusieurs reprises la décision de janvier 1927 considérant comme un abus la diffusion des chants liturgiques de la messe ${ }^{19}$. Par contre, le sermon, la prédication religieuse semblent constituer une utilisation à la fois judicieuse et licite de la radio.

16 Lettre de l'Épiscopat des Pays-Bas, avril 1930, dans Documentation Catholique 523 (1930), col. 1431.

17 Le Bon pasteur, p. 31.

18 Ibid., p. 34.

19 DC 494 (1929), col. 861. 
L'année 1927 marque donc pour la France les débuts d'une présence catholique à la radio tout d'abord avec la retransmission des conférences de Carême du Père Sanson mais surtout avec la prédication radiophonique du $\mathrm{P}$. Lhande que lui-même avait surnommée «L'Évangile par-dessus les toits».

\section{L'Évangile par-dessus les toits}

Le P. Lhande de 1927 à 1934, date à laquelle il est obligé d'arrêter ses radio-sermons, est le prédicateur le plus célèbre de la radio ${ }^{20}$. Sa voix retentit aussi au-delà des frontières, notamment en Amérique latine, où il est invité pour faire une série d'émissions. Il termine chacune de ses conférences par quelques mots en français ou en basque. N'oublions pas qu'il avait consacré à l'émigration basque en Amérique une étude très fournie. Il raconte l'émotion des exilés entendant un écho de la patrie lointaine ${ }^{21}$.

La prédication radiophonique donne au P. Lhande un impact qu'il n'aurait sans doute pas eu sans cela. Évidemment une question essentielle est de savoir si l'utilisation d'un nouveau support change en partie la méthode du prédicateur: $y$-a-t-il des caractéristiques liées au support, le prédicateur parle-t-il de la même façon dans une église ou devant un micro de radio? L'originalité tient-elle à l'émetteur ou plutôt au récepteur démultiplié, non plus les fidèles de telle paroisse, de tel diocèse, mais des catholiques de toute la France? $\dot{A}$ suivre la présentation que la radio fait elle-même de la prédication radiophonique, il semble que les différences soient faibles.

À travers son micro, le $\mathrm{P}$. Lhande traite son immense paroisse invisible de sans-filistes comme un bon curé de ville ou de campagne traite ses fidèles au prône dominical. C'est ainsi que dans le grand cycle des belles solennités que l'Église célèbre depuis Pâques jusqu'à l'époque des pèlerinages, le prédicateur des ondes expose cette fois à ses auditeurs les thèmes liturgiques de la Première communion, de la Pentecôte... Alternant avec ces larges scènes, voici, comme dans des médaillons, des profils ou des figures de saints. Enfin, pour demeurer fidèle à son œuvre capitale et à son sujet de prédilection, l'auteur du Christ dans la banlieue brosse, devant ses chers

20 On trouve aussi Mgr Boucher, les P. Dieux, Gillet, Padé, Carré, Avril et de Pardillez.

21 TSF revue, 8 février 1931. 
paroissiens de Radio, un rapide tableau des nouveaux clochers de la périphérie parisienne vus à vol d'oiseau 22 .

Ainsi, à suivre cette présentation des radio-sermons de 1930 , la prédication radiophonique ne diffère guère d'une prédication classique, les sans-filistes sont assimilés à une immense paroisse invisible. Pourtant, les confidences du P. Lhande lui-même et les nombreuses questions que lui posent ses auditeurs révèlent bien qu'il s'agit d'une prédication particulière.

Il faut d'abord rappeler que la prédication radiophonique du P. Lhande n'est pas la retransmission d'un sermon dit en chaire mais la production d'un discours spécifique qui se veut adapté à ce nouveau moyen de communication. Le P. Lhande a ainsi raconté ses débuts :

L'abbé Gerlier, par mandat du cardinal Dubois, me demanda d'inaugurer une forme nouvelle du ministère de l'Église auprès des âmes. Le 3 janvier 1927, je donne devant le micro de Radiola, situé alors Bd Haussmann, le tout premier sermon porté sur les ondes aux quatre coins du monde ${ }^{23}$.

Le premier sermon avait été prononcé sur le ton de la conversation, le second comme s'il avait été dit en chaire. La correspondance reçue prouva que le premier sermon avait été le plus apprécié. Le P. Lhande disait: "Il faut parler d'ami à ami, discrètement au cœur, sobrement à l'esprit ${ }^{24}$. Il est très conscient que faire un sermon à la radio demande de trouver un style nouveau. Lui-même témoigne:

Il fallait donc trouver un genre nouveau de prédication, spécialement adapté par le ton, le style, la durée, aux conditions très particulières de la radiophonie et à un public infiniment varié, comprenant toutes les bigarrures de la pensée, de la culture, du rang ${ }^{25}$.

À cette situation répondit le «radio-sermon», c'est-à-dire une «causerie religieuse brève, vivante, adaptée, plus parlée que déclamée, émise en une heure favorable à l'intimité du foyer ${ }^{26}$. Les auditeurs s'interrogent sur les difficultés de cette prédication faite dans un micro et le $P$. Lhande répond à leur curiosité :

22 Fiche de présentation pour les radio-sermons de mai à juillet 1933 (ASJV L5/1).

23 Cité sans référence dans E. GILBRIN: "De l'Évangile par-dessus les toits à la paroisse des ondes », C.H.A., avril 1983. $\mathrm{n}^{\circ} 107$, p. 5.

24 Ibid.

25 L'Évangile par-dessus les toits. Radio-sermons série 1927, p. 14-15.

26 Ibid. 
Comment nous dit-on pouvez-vous parler avec conviction et même avec émotion, à un auditoire que vous n'apercevez point? Cela doit être si déconcertant de s'adresser à une foule invisible [...] En vérité dès qu'apparaît sur le transparent lumineux le mot solennel Silence! vous êtes tous là... Dans la seconde qui s'écoule entre l'avis du speaker et mon signe de croix, je vous devine, je vous aperçois, je vous résume en un éclair: les grands, les petits, les riches, les pauvres, les petits bourgeois, les ouvriers, les malades [...] Croiriezvous que la seule perspective de cette rencontre mystique me donnc, dès le dimanche matin, une sorte de petite fièvre, comme un trac fait d'angoisse et de joie 27 .

À ses auditeurs qui s'étonnent: «mais alors vous lisez vos causeries devant le micro?» Il répond:

Oui, je lis et je ne lis pas. Les feuillets sont là, étalés sur le pupitre, devant cette mystérieuse petite boîte d'acier suspendue à son soutien métallique [...] Mes yeux vont de la page blanche au sombre micro. $\grave{A}$ tout moment, $j$ 'abandonne mon texte. Je coupe ma phrase; je la hache d'interjections familières. Je vous dis: "Me comprenezvous?» Et vous me comprenez. Je vous dis: «Vous pleurez?» Et vous pleurez. Quelle chaire au monde peut donner cette puissance de communication ?28

À lire les causeries religieuses ou les sermons du P. Lhande, on est frappé par la liberté de ton, voire la familiarité en début, en fin ou en marge des sermons. Ainsi commence-t-il un sermon à radioLuxembourg intitulé «le rosaire de Pâques».

Un tout petit mot avant de commencer mon sermon. Pour vos étrennes je vais vous offrir ... un œuf de Pâques, un œuf à surprise ... Pas un œuf en chocolat, avec des bonbons dedans comme pensent sans doute en m'écoutant mes petits amis sans-filistes de huit à douze ans, mais un œuf de Pâques d'un genre nouveau: un œuf radio-sermon... Oui, je sais, l'expression n'est pas très liturgique. Mais nous ne sommes pas à l'Église, nous sommes à l'auditorium de Radio-Luxembourg, dans un grand studio [...] Nous ne sommes donc pas tenus au recueillement et à la gravité que nous commanderait la chaire chrétienne et avons droit, dès lors, à notre petite liturgie à nous ${ }^{29}$.

27 P. LHANDE, "La causerie religieuse du dimanche»: rapport au Congrès catholique du cinéma et de la radio, sans date (ASJV L5/1).

28 Rapport au Congrès catholique du cinéma et de la radio (ASJV L5/1).

29 Sermon du ler avril 1934 (ASJV L5/1). 
De son aveu même le $\mathrm{P}$. Lhande s'octroie plus de liberté que lors d'un prêche à l'église, non seulement le lieu est moins solennel mais en outre il s'adresse moins à un vaste auditoire qu'à chaque individu en particulier. Le ton est à la fois plus libre et intimiste. Il interpelle ses auditeurs notamment lors d'une prédication de carême centrée sur la figure de sainte Thérèse de Lisieux:

Je voudrais que vous ayez sous les yeux sa douce image, comme je l'ai moi-même là devant le micro [...] Je vous prie donc vous tous qui avez à portée de main une de ces gravures de la poser pendant mon entretien près de votre haut-parleur ... Geneviève, va la chercher dans ta chambre ... Marguerite, dans ton paroissien ... Jacquot, Dédé, Marcel, Pierre, sur votre cheminée ... Je vous attends ... Là ... c'est fait? Je commence ${ }^{30}$.

Il les interpelle passant au tutoiement pour bien marquer qu'il s'adresse à chacun d'eux, égrenant des prénoms. L'intimité que le P. Lhande témoigne à ses auditeurs est nourrie par la connaissance qu'il a d'eux grâce à une volumineuse correspondance.

Ce retour après le discours qui permet de savoir comment a été reçue la prédication est aussi une des originalités de ce type de communication.

\section{La réception des radio-sermons}

Le succès est immédiat, le $\mathrm{P}$. Lhande tente d'expliquer cet extraordinaire aimant de la parole ailée et observe «que l'auditeur moyen se trouve dans les meilleures conditions morales pour profiter d'une bonne parole, au calme, chez soi, le plus souvent aucunement extériorisé ni distrait, l'esprit entièrement tendu vers le haut-parleur \$1. $^{31}$ La réception semble largement dépasser le cadre des seuls catholiques pratiquants, encore que le nombre limité de postes dans la population française demande de relativiser l'impact des émissions radiophoniques. Le P. Lhande affirme que «les juges compétents estiment au chiffre d'un million le nombre des sansfilistes qui écoutent habituellement notre causerie dominicale $\${ }^{32}$.

30 Le Bon pasteur, p. 50

31 L'Évangile par-dessus les toits. Radio-sermons série 1927, p. 18

32 Chiffre donné lors du radio-sermon du $1^{\mathrm{er}}$ avril 1934 sur Radio-Luxembourg (ASJV L5/1). 
Il est à noter aussi que bon nombre de catholiques vivant loin des grandes villes sont privés des secours de l'éloquence. Une auditrice belge écrit: "Nous qui vivons dans un petit village de campagne sommes privées de beaux sermons, nous sommes ainsi largement compensées, comme cela fait du bien $\rangle^{33}$.

La Fédération nationale catholique, qui a créé en 1927 une association d'auditeurs sous le nom de radio-familles ${ }^{34}$, accueille avec enthousiasme les sermons radiodiffusés du P. Lhande et fait une recension très élogieuse de leur publication.

L'une des plus grandes révélations scientifiques et religieuses de l'année 1927 a été sans nul doute celle du pouvoir que possède la TSF de traduire et de révéler presque à l'infini les enseignements et les émotions de la foi. Du premier coup, la radiophonie s'est affirmée triomphante dans ce mode nouveau de transmission de la pensée supérieure [...] Ses radio-sermons ont obtenu le suffrage de tous les sans-filistes qui ont exprimé aussitôt le désir de les posséder pour les relire à loisir ${ }^{35}$.

En effet, la parole du P. Lhande se transforme en écrit et la publication de ses sermons est toujours un succès. Parfois même, elle est conservée sous la forme d'un disque. Lors de son sermon à RadioLuxembourg, le Père expose ce qu'il attend de ce moyen de diffusion.

Songez que cette même causerie, reproduite sur disque, seulement à 10000 exemplaires, tournée en famille ou en séance publique seulement 30 fois devant une moyenne de seulement 5 auditeurs nouveaux est donc répétée 300000 fois et atteint un million et demi de personnes. Quelle prodigieuse multiplication de la parole de Dieu! Bien des fois j'ai entendu formuler un regret: «Je ne puis vous entendre, hélas! Je n'ai pas la TSF !» Soit! Mais qui n'a pas à sa portée un simple phonographe? 36

C'est la société Lumen spécialisée dans la musique religieuse et les vieilles chansons françaises qui se charge de l'impression du disque ${ }^{37}$. Un curé de campagne témoigne:

33 Lettre du 20 octobre 1929 (ASJV L5/5.4).

34 En 1930, cette association est transférée au CCC (Comité catholique du cinéma) où elle prend le nom d'Antennes familiales.

35 Credo, avril 1928.

36 Sermon «Le rosaire de Pâques» dit à Radio-Luxembourg le $1^{\text {er }}$ avril 1934 (ASJV L5/1).

37 Disque vendu au prix de 25 francs. 
Je viens vous remercier pour mes paroissiens d'avoir bien voulu donner un disque : il est très apprécié chaque fois que je le donne à mes auditeurs dans les réunions que je fais dans mes poussières de villages. Une petite auto me permet d'atteindre mon monde et de transporter de quoi faire autre chose que demander des nouvelles de tout le monde, parler de la pluie et du beau temps et ... demander le denier du culte. Si vous pouviez nous donner d'autres disques de sermons... ${ }^{38}$.

La prédication radiophonique n'a-t-elle alors que des avantages? Un pasteur chargé de faire un article sur les sermons radiodiffusés pose au P. Lhande quelques questions pertinentes.

En quoi considérez-vous comme nécessaires les sermons par radio? $\mathrm{Ne}$ croyez-vous pas que deux objections peuvent se présenter à ce sujet: les fidèles qui écoutent cela au milieu d'annonces et d'avis divers sont-ils bien préparés à s'édifier? Le culte en pantoufles me semble souvent une injure et un manque de respect; de plus, il manque à ces services l'influence qu'on retire d'une communion fraternelle dans la prière et sous l'influence du lieu saint dans lequel on célèbre le culte ${ }^{39}$.

Nous n'avons malheureusement pas la réponse du $\mathrm{P}$. Lhande à ces questions. Mais l'ensemble de ces interventions indique bien qu'il parle avant tout pour des personnes qui ne peuvent aller fréquemment à l'Église, malades ou personnes isolées. D'autre part, le cardinal Dubois a obtenu que chaque causerie religieuse soit précédée et suivie d'une minute de silence pour faciliter justement la transition et le recueillement. Enfin pour la plupart des catholiques, l'écoute de la radio n'empêche aucunement d'aller à l'Église le dimanche matin. Mais la réponse consiste sans doute dans le succès même des causeries du P. Lhande.

Les lettres conservées par le $\mathrm{P}$. Lhande permettent de comprendre ce qui fait la spécificité de ces radio-sermons.

C'est un fait curieux que, au moins pour moi, un sermon entendu dans ma petite chambre d'un prédicateur invisible touche bien le cœur comme si toute distance est ( sic) anéantie et nous sommes tout près de l'invisible. Le résultat est beaucoup plus grand que celui produit par un sermon qu'on entend de la façon ordinaire où l'on voit le prédicateur ${ }^{40}$.

38 Lettre de l'abbé DeLAGE, curé de Jourgnac, 30-10-1930 (ASJV L5/1).

39 Lettre du pasteur LOCKERT de la paroisse de Puteaux, 5 août 1927 (ASJV L5/1).

40 «Mes impressions» par Sophie O'BRIEN (ASJV L5/1). 
Cette proximité plus grande vient justement du fait que le prédicateur est invisible et que rien ne vient s'interposer entre sa voix et celui qui écoute.

Les auditeurs du P. Lhande sont trop nombreux et divers, c'est ce qui fait l'originalité de cette prédication, pour pouvoir en dresser un tableau. D'ailleurs ils ne nous sont connus que par leur correspondance et il n'est pas sûr que ceux qui écrivent soient représentatifs de l'ensemble beaucoup plus vaste. Ce qui est sûr, c'est que parmi ceux qui écrivent au P Lhande, les malades, les personnes âgées et toutes celles qui viennent d'être éprouvées par un deuil sont particulièrement nombreuses. Il s'agit plutôt, mais pas toujours, de personnes modestes. Le père jésuite reçoit aussi de nombreuses lettres d'enfants généralement accompagnées de «piécettes» pour les pauvres.

Inconnus de cette voix, des auditeurs se confient, se confessent même dans une franchise indubitable. Il insère d'ailleurs de nombreux extraits de ces lettres à la fin des volumes de radio-sermons, tout en préservant bien sûr le total anonymat de ses correspondants. On trouve bien évidemment dans la prédication du P. Lhande une place toute particulière concernant les ouvriers. En mars 1928, il consacre son radio-sermon lu à Londres au "Christ chez les ouvriers», aux "pauvres dans l'évangile». La prédication du P. Lhande repose sur la description et l'injonction: description de l'afflux des masses populaires vers Paris et de leurs conditions de vie, injonctions aux auditeurs.

Dites vous bien que ces travailleurs, ces pauvres sont votre plus proche prochain et leur évangélisation ou leur soulagement votre plus impérieux devoir [...] Employez-vous donc de tout votre pouvoir, les uns par leur ministère, les autres par leur charité, à donner le Christ à ces frères déshérités qui ne le possèdent point ${ }^{41}$.

Les figures des saintes Thérèse de Lisieux, Germaine de Pibrac et Jeanne d'Arc fournissent le thème de la prédication du carême de 1927. Celle de 1928 est consacrée au récit de la résurrection de Lazare, entrecoupé par des interpellations aux auditeurs qui eux aussi doutent ou qui reprochent à Dieu de ne pas avoir exaucé leurs

41 Le Bon pasteur, p. 44. 
prières. La cananéenne doit illustrer la miséricorde du Christ à l'égard d'une étrangère ${ }^{42}$.

Les lettres d'auditeurs ont été conservées par le P. Lhande et constituent un témoignage remarquable sur la réception de cette prédication radiophonique. À la lecture de ces innombrables lettres, il apparaît que la parole du $\mathrm{P}$. Lhande convertit, console et incite à la charité.

Si le but premier de toute prédication est de convertir, celle du P. I hande paraît réussie. Des dizaines d'auditeurs témoignent de leur conversion par TSF, ou du retour à Dieu d'eux-mêmes ou des membres de leur famille: «vous l'avez converti par TSF».

Je suis l'un de vos paroissiens de TSF et je viens vous annoncer ma conversion ... J'ai grandi entre des prières sans réponse et des larmes sans consolation. Depuis deux ans, je ne priais plus. J'ai acheté un poste de TSF pour me distraire et n'ai pas perdu un seul de vos sermons. Vous m'avez conquis ... Pourquoi ... Je ne sais pas ... Mais dans votre voix, il y a du divin. J'ai donc fait mes Pâques cette année ${ }^{43}$.

Les auditeurs lui confient leurs doutes. Telle cette habitante de l'Allier: "Je me demande parfois si j'ai toujours la foi, mais si j'en ai encore une étincelle je crains bien de la perdre tout à fait si je ne trouve personne pour m'éclairer ${ }^{44}$. Plusieurs donc témoignent d'un retour à la foi après avoir écouté les sermons du P. Lhande à la radio. La conversion est sans doute un des buts premiers de la prédication, la prédication radiophonique permet de ramener à la foi des personnes qui n'entrent plus ou pas dans une église: chez eux, par curiosité au minimum, ils écoutent, et là saisis par une voix, des propos qui touchent les personnes humbles, ils deviennent des fidèles de cette prédication avant de devenir de fidèles chrétiens. La radio jouit de l'avantage d'une démarche minimale que l'on peut imaginer plus facile qu'entrer à l'église, ou aller à la messe.

Le succès de la parole passe dans les actes, la prédication radiophonique démultiplie l'écho de l'appel à l'assistance. Au cours de l'hiver 1929 particulièrement rigoureux, les ouvriers du bâtiment sont réduits au chômage technique. Le P. Lhande demande à ses audi-

42 Prédication du carême de 1928.

43 Lettres conservées dans quatre cartons aux ASJV, L/5/5.

44 Lettre du 21 avril 1929 (ASJV L5/5.1). 
teurs d'envoyer des secours. Ils arrivent alors en quantité telle qu'ils nécessitent l'aide de plusieurs Euvres de charité pour en faire la distribution. Le P. Lhande a publié des dizaines d'extraits de lettres de reconnaissance. Il conclut certes à l'efficacité de l'émission radiophonique mais surtout à celle de la charité privée. Le 9 mars 1930, il demande d'aider les inondés du Midi et les auditeurs répondent encore si nombreux que les sœurs de Saint-Vincent de Paul font la navette durant 6 jours pour désengorger le siège des Études: «Seul l'accent de la charité privée, de la charité chrétienne peut exercer sur les hommes une action aussi intense et aussi large $\gg^{45}$.

Il est tout à fait remarquable que la très grande majorité des lettres reçues par le P. Lhande ${ }^{46}$ soient accompagnées d'une obole ou d'un colis pour secourir les pauvres.

La prédication radiophonique connaît certaines contraintes du fait de la diversité maximale des publics. Elle se veut d'autant plus prudente qu'elle se sait menacée par les tenants du camp laïque. Le P. Lhande donne quelques conseils aux orateurs populaires de la radiophonie :

Éviter toute attaque aux théories qu'on ne partage point, observer toujours un ton de suprême réserve et de modération, entrer en contact avec les humbles, les petits qui constituent l'auditoire le plus attentif et le plus émotif; ne point se perdre dans un dogmatisme abstrait mais parler d'actualité : bref appliquer à ce ministère si neuf le code principal de l'éloquence d'après saint Augustin: "La grande loi de celui qui parle c'est le bien de celui à qui il parle $»^{47}$.

La maison d'édition Spes publie avec constance tous les sermons radiodiffusés du P. Lhande: les Radio-Sermons de 1927 tirés à 11500 exemplaires sont épuisés de même que ceux de 1928 sous le titre de L'Evangile par-dessus les toits ${ }^{48}$ ou Le Bon pasteur ${ }^{49}$.

45 «La TSF au secours des pauvres», mars 1929.

46 Lettres conservées dans quatre cartons aux ASJV, L/5/5.

47 Propos prêtés au P. Lhande par un article du Correspondant, 25 janvier 1930.

48 Les chiffres des éditions donnés en 1930 témoignent d'un succès croissant. Le total des éditions vendues, tous titres confondus, s'élevant à 381176 exemplaires (ASJV Ll/1, dossier $\mathrm{n}^{\circ}$ 5).

49 Liste complète des radio-sermons publiés: L'Évangile par-dessus les toits, 1927 ; Le bon pasteur, 1928; Les pauvres dans l'Évangile, 1929; Les Béatitudes, 1929; De Bethléem au Golgotha, 1930; Le Pater, 1930; La France missionnaire, 1931; L'Évangile de la douleur, 1932; L'Évangile par-dessus les frontières, 
La prédication radiophonique ne s'arrête évidemment pas avec le P. Lhande. Son aphasie consécutive à une attaque l'oblige à cesser ses sermons en 1934. Il est alors remplacé par un dominicain le P. Roguet. Dix ans après les débuts du P. Lhande, les premières messes sont diffusées à la radio, celle célébrée à la Madeleine pour la Pentecôte de 1937 et celle du cardinal Gerlier à Radio-Lyon le 23 octobre $1938^{50}$.

Le $P$. Lhande a effectivement joué un rôle de pionnier en assumant pour la première fois en France une prédication radiodiffusée. Ses succès en font rapidement un exemple, il plaide la cause de la radio auprès du cardinal Faulhaber, archevêque de Munich qui ne lève l'interdit d'émettre des conférences sacrées par radio qu'en 192951. En 1930, il donne 18 conférences en un mois à la radio de BuenosAires, 8 à Montevideo; à Valparaiso il tente l'évangélisation de la banlieue noire los Cerros, au moyen d'une vingtaine de postes répartis en plein air.

Le P. Lhande a compris qu'à ce nouveau moyen de communication, il fallait adapter de nouvelles formes de prédication et instaurer un dialogue permanent avec les auditeurs. Le 12 février 1931, pour la première fois, la radio retransmet un message de Pie XI.

Corinne BonafouX-VERRAX Université de Savoie, Chambéry

1932; Paraboles, 1933; Carillons de fête, 1933; Petites béatitudes pour jours d'épreuves, 1934.

50 «De l'Évangile par-dessus les toits à la paroisse des ondes», $C H A$, avril 1983, p. 6

51 Carillons de fête, p. 249. 